\title{
LES ANOMALIES CHROMOSOMIOUES DANS LES TUMEURS SOLIDES : QUELLE SIGNIFICATION ?
}

\section{Bernard Dutrillaux}

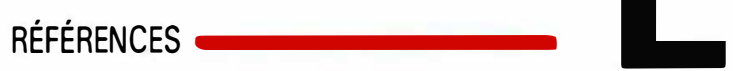

a cytogénétique des tumeurs solides a connu un nouvel essort au cours de ces dernières années. Il s'agissait de transposer aux cancers ce qui venait d'être découvert dans la leucémie myéloïde chronique et le lymphome de Burkitt, c'est-à-dire la mise en évidence d'une dérégulation d'oncogènes par effet de position dominant, consécutif à une translocation spécifique $t(9 ; 22)$ pour l'une et $t(8,14)$ pour l'autre [1,2].

La recherche d'autres translocations spécifiques semblait donc une voie d'approche toute tracée, qui a porté ses fruits pour un certain nombre d'hémopathies. Il n'en a malheureusement pas été de même pour la très grande majorité des cancers humains, qui touchent les cellules épithéliales du sein, du côlon, du poumon, de la peau, etc. Aucun remaniement chromosomique, pas plus d'ailleurs que de lésion génétique, ne les caractérise vraiment. Cela ne constitue pas pour autant un constat d'échec, mais indique que les idées de départ étaient trop simplistes.

Quelle interprétation peut-on donc donner aux anomalies chromosomiques multiples observées dans presque tous les cas de cancer? Leur complexité et leur nombre élevé, dans une même cellule, pourraient laisser croire qu'elles ne sont que le témoin, sans importance biologique réelle, des dérèglements affectant tout tissu cancéreux. Toutefois, une analyse moins sommaire montre que certaines anomalies, bien que non systématisées, sont très récurrentes dans un type de cancer donné. Il est donc peu probable qu'elles surviennent ou se fixent par hasard.

L'atteinte d'un segment chromosomique donné n'est cependant pas univoque, car de nombreux remaniements différents sont susceptibles d'intervenir. Quant aux points de casure chromosomiques, qui pourraient attirer l'attention sur un segment génomique précis, ils sont généralement variables, et touchent le plus souvent l'hétérochromatine juxta-centromérique, composée d'ADN répétitifs non codants, dont il est peu probable que la recombinaison puisse induire une dérégulation génique. En fait, ce qui semble important, c'est le déséquilibre chromosomique, par gain ou perte d'un segment, d'un bras, voire d'un chromosome entier. Dès lors que ces anomalies sont analysées quantitativement, les profils de déséquilibres apparaissent très caractéristiques.

Par exemple, un remaniement du chromosome 1 aboutira le plus souvent à une duplication du bras long dans les cancers du sein et de l'utérus et, plus rarement, à la perte du bras court, ou aux deux, mais jamais à la combinaison inverse. Dans le cancer du côlon, seule la délétion du bras court s'observera, dans environ $50 \%$ des cas. Pour de nombreux autres chromosomes, les pertes sont plus fréquentes que les gains, de sorte qu'une réduction progressive du caryotype survient. Ainsi, un cancer du sein ou du côlon évolué peut perdre plus de $20 \%$ de son génome haploïde, alors qu'il n'en duplique guère plus de $10 \%$

Plus la cellule cancéreuse se déséquilibre en perdant des chromosomes, plus elle a tendance à subir une endoréplication, d'où un gain de matériel chromosomique brutal, mais avec conservation des déséquilibres. Dans la cellule hypotétraploïde, qui en résulte, le processus de pertes chromosomiques individuelles s'accélère, de sorte que le nombre de chromosomes redescend progressivement autour de la cinquantaine. Ainsi, de nombreux cancers du sein, du poumon ou du côlon à 50-60 chromosomes sont passés par la tétraploïdie. On comprend que cela aboutisse à un profond changement de la balance allélique : les pertes sont nombreuses, et souvent les deux chromosomes res- 
tants ont la même origine parentale. Ce processus explique les nombreuses descriptions, dans la littérature, des pertes de polymorphisme dans ces tumeurs, et incite à relativiser l'importance de chaque cas particulier.

Les chromosomes sexuels suivent une évolution semblable, compliquée par le fait qu'un seul est habituellement transcrit. Ainsi, la perte de l'Y dans les tumeurs mâles, et de l'X inactivé, de réplication tardive, dans les tumeurs femelles, est très fréquente, et est souvent suivie par la duplication de l'X actif, de réplication précoce [3]. Pour ces chromosomes, le remodelage observé peut difficilement être attribué à l'avantage que confererait la conservation de tel allèle par rapport à tel autre. En effet, seuls les allèles initialement transcrits de l'X actifs sont conservés, et dupliqués. Le dosage génique doit donc avoir aussi son importance, à côté du déséquilibre allélique.

Dès le début des années 1980, l'intérêt du déséquilibre allélique a clairement été mis en évidence par Cavenee et al. [4] dans le rétinoblastome (Rb). Depuis plusieurs années, on savait qu'une délétion du chromosome 13 s'observait parfois à l'état constitutionnel dans cette maladie, et bien que le gène $R b$ ne fût pas encore connu, il fut possible de montrer que différentes combinaisons chromosomiques aboutissaient toutes à la perte d'un petit segment du 13 , et donc au passage à l'hémizygotie ou à la nullizygotie. Ceci permettait de conclure au caractère récessif de la mutation du gène $R b$. Actuellement, toutes les interprétations ont été précisées, et le gène $R b$ est devenu l'anti-oncogène type : gène codant pour une protéine dont la fonction contrôle d'autres protéines à activité oncogène. Ce concept ne fut pas facilement admis, initialement, car il était établi, à l'époque, que le cancer résultait nécessairement de l'activation d'oncogènes, ras en particulier. Le même facteur de mode joue maintenant en faveur des antioncogènes. Doit-on pour autant interpréter toutes les pertes chromosomiques (ou alléliques) comme autant de pertes de fonctions antioncogènes? Rien n'est moins sûr.
De plus, le concept d'anti-oncogène doit être pris au sens large, comme le montre l'exemple de la p53. Le gène était initialement considéré comme un oncogène, car la protéine p53 était retrouvée en abondance dans de nombreux cancers et lignées de cellules transformées. Pourtant, la délétion du bras court du chromosome 17 , qui porte ce gène, était décrite dans $80 \%$ des cas de cancers du côlon, bien qu'il y ait une forte expression de la p53 dans ces tumeurs. Ce paradoxe s'explique par le fait que la surexpression est consécutive à une mutation [5] : le gène p53 normal aurait donc bien une fonction anti-oncogène tandis que le gène muté aurait une fonction oncogène. Il est même possible qu'un autre gène du bras court du 17 contrôle l'expression de p53 [6]. Ainsi, la perte de l'allèle normal et la surexpression de l'allèle restant, anormal, vont bien dans le même sens, et surviennent évidemment l'un après l'autre au cours de la progression tumorale. Bien qu'on suspecte qu'ils soient nombreux et que plusieurs " candidats" aient été proposés, dans le cancer du côlon (gène $D C C, \mathrm{~m} / \mathrm{s}$ $n^{\circ} 3$, vol. 6, p. 292), la neurofibromatose de type 1 (gène $N F-1, m / s n^{\circ} 8$, vol. 6, p. 815) et la tumeur de Wilms (gène $W T ; \mathrm{m} / \mathrm{s} n^{\circ} 5$, vol. 6, p. 464), aucun autre anti-oncogène n'est connu aussi bien que p53 et p105 Rb Mais peut-être arrivera-t-on à conclure que beaucoup de gènes "de ménage" ont une fonction antioncogène. Actuellement, les pistes, les plus sérieuses orientent les recherches sur le bras court du 1 (cancer du côlon et du sein), le bras court du 11 (cancers du rein et du foie) et le bras court du 3 (cancer du poumon) et le bras long du 17 dans les cancers du sein et de l'ovaire avec agrégation familiale [7] [Feunteun $\mathrm{J}$ et Lenoir G, communication personnelle].

Aussi fréquentes que puissent être certaines anomalies chromosomiques, aucune n'est constante dans une tumeur donnée. Lorsque l'on parvient à reconstituter l'évolution clonale d'un certain nombre de tumeurs d'un même type, on voit que même les anomalies les plus fréquentes peuvent survenir tardivement, dans un sous-clone. Toutes les modifications chromosomiques (et donc leurs conséquences moléculaires) sont donc des événements facultatifs de la progression tumorale.

Une tumeur donnée se traduit par un seul, ou un nombre réduit de profils chromosomiques :

- perte des chromosomes $17 \mathrm{p}(\mathrm{p}=$ bras court), 18, 1p, 8p, 4, 14, 10q ( $\mathrm{q}=$ bras long), 15, 21, 5q, et gain de l'X précoce et des 13,20 et $8 \mathrm{q}$ dans la majorité des cancers colorectaux pour la forme monosomique, qui se distingue de la forme trisomique (20\% des cas) qui a peu de délétion mais dans laquelle on constate des gains d'autres chromosomes, 7 et 12 principalement [8] ;

- perte des 17p, 13, 8p, 11p, 11q, $16 q, 6 q$ et gain des $1 q$ et $8 p$ dans le cancer du sein [9];

- gain des 1q, 10, 2, 7 et 12 dans le cancer de l'endomètre ;

- perte du 3p, 5, 8p, 10p, 13 et gain de $1 \mathrm{q}, 3 \mathrm{q}$ et 7 dans les cancers pulmonaires ;

- perte de 9p, 10, 22 et moins souvent $6 \mathrm{q}, 1 \mathrm{q}, 14$ et gain de 7 dans les gliomes, etc.

Ce qui caractérise un type de tumeur, ce n'est donc pas une, mais un ensemble d'anomalies chromosomiques. De plus, une même anomalie, comme la délétion du bras court du chromosome 17 pourra être partagée par des tumeurs aussi différentes que les cancers du sein ou du côlon, et même la leucémie myéloïde chronique, où elle survient souvent au cours de l'acutisation. Les gains de 1q et de 7 s'observent dans de nombreux cancers solides. Prenons un autre exemple, avec le chromosome 13. Sa délétion, aboutissant à la perte de fonction du gène $R b$, est très probablement l'événement initial du rétinoblastome. Ce chromosome est déficient dans un fort pourcentage (environ $50 \%$ ) des cancers pulmonaires et mammaires. Par ailleurs, l'expression du gène $R b$ est supprimée dans un pourcentage comparable de cas de ces tumeurs. Les deux événements sont probablement liés, et la perte de la fonction antioncogène ne serait plus ici qu'un événement facultatif de la progression tumorale. Pour le gène $R b$, comme pour celui de la p53, il est intéres- 
sant de savoir si la mutation de l'allèle restant précède ou suit la perte de l'allèle normal, c'est-à-dire la délétion chromosomique. L'importance de cette question réside en ce qu'elle débouche directement sur la compréhension des facteurs de sélection vers tel ou tel profil chromosomique. L'hypothèse que les gènes $R b$ et $p 53$ sont les facteurs de sélection est sans doute trop réductionniste. N'oublions pas en effet que l'hémizygotie favorise considérablement l'expression d'emblée de toute mutation récessive ( $\mathrm{p}$ au lieu de $2 \mathrm{p}^{2}$ ), et que la perte chromosomique peut donc précéder la mutation du gène d'intérêt.

C'est précisément la recherche de ces facteurs de sélection qui a orienté une bonne part de nos activités. Leur existence est difficile à démontrer, et nos résultats ne sont pas encore que fragmentaire. Dans le cancer colorectal, les délétions touchent de nombreux gènes codant pour la synthèse endogène des nucléotides alors que les gains touchent des gènes de la synthèse exogène, de récupération. La balance entre ces deux voies penche plus que pour la moyenne des tissus en faveur de la voie exogène dans la muqueuse colique normale. Ce déséquilibre devient parfois très fort dans les adénocarcinomes. Nous avons pu montrer qu'il est typique de la forme monosomique définie cidessus [10]. Ainsi, la pression métabolique contribuerait à déséquilibrer progressivement le caryotype en fonction de nouveaux impératifs de transcription. Dans les cancers de l'endomètre, les gains touchent de façon très préférentielle les segments qui portent les gènes de la glycolyse, activité qui est effectivement fortement réactivée dans les cellules de ce cancer. Dans les gliomes, les pertes chromosomiques affectent des gènes impliqués dans le métabolisme de l'adénylate, tandis que dans plusieurs autres cancers, comme celui du sein, les délétions atteignent souvent des segments chromosomiques porteurs de gènes de détoxification, comme la superoxyde dismutase 2 du bras long du 6 et la catalase du bras court du 11.

Ainsi, toute une cascade d'événe- (lente ?) transformation d'un tissu normal en un tissu cancéreux. Pour ce qui concerne les cancers solides, les anomalies chromosomiques jalonneraient les étapes de la progression tumorale, et ne seraient que rarement parmi les événements les plus précoces. Elles surviendraient à la suite de mutations (pertes de fonctions réparatrices de l'ADN) ou d'autres modifications génomiques (changements de méthylation de l'ADN). La ségrégation des chromosomes remaniés serait ensuite sous la dépendance de facteur sélectifs puissants, parmi lesquels les déviations métaboliques auraient un rôle important. Il en résulterait les déséquilibres chromosomiques non aléatoires, qui auraient eux-mêmes pour conséquence de multiples déséquilibres alléliques. Parmi ceux-ci, les passages à l'hémizygotie permettrait l'expression de mutations récessives, dont une partie correspondrait à des pertes de fonctions anti-oncogènes. Les gains de chromosomes suggèrent fortement que les effets de dosage génique existent et ne se limitent pas aux phénomènes d'amplification. Leur importance est très probablement sous estimée par le monde scientifique actuel, mais il faut reconnaître qu'à part la possibilité d'une sélection en relation avec les déviations métaboliques, aucune hypothèse n'explique leur existence.

Finalement, plus que toute autre approche, la cytogénétique des tumeurs solides humaines a bien montré le caractère multifactoriel des désordres génétiques, et le fait que ces anomalies surviennent successivement au cours de la progression tumorale

\section{TIRÉS A PART}

B. Dutrillaux. 